\title{
Alteration and recovery of the antioxidant system induced by sub-chronic exposure to microcystin-LR in mice: Its relation to liver lipid composition
}

\author{
Daniela Sedan $^{\mathrm{b}}$, Darío Andrinolo ${ }^{\mathrm{a}, \mathrm{b}, *}$, Luis Telese ${ }^{\mathrm{b}}$, Leda Giannuzzi ${ }^{\mathrm{a}, \mathrm{b}}$, María J.T. de Alaniz ${ }^{\mathrm{c}}$, \\ Carlos A. Marra ${ }^{\mathrm{c}}$ \\ a CIDCA (Centro de Investigación y Desarrollo en Criotecnología de Alimentos), Universidad Nacional de La Plata UNLP, CCT-La Plata CONICET, Argentina \\ ${ }^{\mathrm{b}}$ Toxicología, Facultad de Ciencias Exactas, Universidad Nacional de La Plata UNLP, La Plata, Argentina \\ ${ }^{\mathrm{c}}$ INIBIOLP (Instituto de Investigaciones Bioquímicas de La Plata), Cátedra de Bioquímica y Biología Molecular de la Facultad de Ciencias Médicas de la Universidad \\ Nacional de La Plata UNLP, CCT-La Plata CONICET, Argentina
}

\section{A R T I C L E I N F O}

\section{Article history:}

Received 13 May 2009

Received in revised form 12 August 2009

Accepted 14 August 2009

Available online 20 August 2009

\section{Keywords:}

Microcystin

Lipids

Oxidative stress

Antioxidant enzymes

Hepatic damage

\begin{abstract}
A B S T R A C T
The effects of MC-LR on antioxidant system in liver and kidney and its effects on hepatic lipid composition after prolonged exposure to sublethal doses of microcystins (MCs) were studied in mice. Mice were treated i.p. with $25 \mu \mathrm{g}$ of MC-LR/kg body weight or saline solution every 2 days for a month (inflictive stage), then progression or recovery was studied for 1 and 2 months of wash-out. During the inflictive stage, MC-LR-induced oxidative damage and significant changes in liver lipids of treated mice were compared with control mice.

A clear dependence of the enzyme defense system was demonstrated with reduced glutathione and $\alpha$-tocopherol availabilities and a concomitant elevation in NOx production.

Sub-chronic MC-LR toxicosis produced alterations in lipid components that included a decreased EFA/non-EFA, SFA/PUFA, and n-3/n-6 ratios all of which exhibited a pattern of slow recovery during the recovery periods.
\end{abstract}

(c) 2009 Elsevier Ltd. All rights reserved.

\footnotetext{
Abbreviations: CAT, catalase; DBI, double bond index; GPX, glutathione peroxidase; GSH-Tr, glutathione S-transferase; ECHO, cholesteryl ester; EFA, essential fatty acids; GSH, reduced glutathione; i.p., intraperitoneal; GSSG, oxidized glutathione; GSH-Rd, glutathione reductase; LPO, lipid peroxides; MCs, microcystins; MC-LR, microcystin-LR; NEFA, non-esterified fatty acids; NL, neutral lipids; Non-EFA, non-essential fatty acids; NOx, nitrate plus nitrite levels; NOS, nitric oxide sinthase; PhtChol, phophatidylcholina; PhtEth, phosphatidylethanolamine; PL, polar lipids PUFA, polyunsaturated fatty acids; ROS, reactive oxygen species; SFA, saturated fatty acids; SOD, superoxide dismutasa; TAG, triacylglycerides; $\mathrm{TL}$, total lipids.

* Corresponding author. Área de Toxicología, Facultad de Ciencias Exactas, Universidad Nacional de La Plata, CONICET, 47 y 115, 1900 La Plata, Argentina. Tel.: +54 221 4250497; fax: +54 221 .

E-mail address: dandrinolo@yahoo.com (D. Andrinolo).
}

\section{Introduction}

MCs are considered the most common group of cyanotoxins in estuaries, ponds and water reservoirs associated with acute, even lethal, toxicity in various species of domestic animals, wildlife and humans (Falconer, 1999; Jochimsen et al., 1998). Microcystins are cyclic heptapeptides consisting of seven amino acids, including several D-amino acids and two unusual amino acids, namely the Nmethyldehydroalanine (Mdha) and hydrophobic $\beta$-amino acid, 3-amino-9-methoxy-2,6,8-trimethyl-10-phenyldeca4,6-dienoic acid (Adda). Microcystin-LR (MC-LR) is the most frequent, toxic and commonly studied isoform of these hepatotoxins (WHO, 2004).

The mechanisms involved in chronic intoxication may also be responsible for the increased incidence of primary liver cancer in humans living in some areas of China 
Chronic exposure to sublethal concentrations of MCs is the most common intoxication in wild and domestic animals and humans (Ueno et al., 1996; Zhao et al., 2009).

It is known that the major route to MCs exposure is through water intake. The stomach absorbs MC-LR to some degree, but the main route of absorption is considered to be the small intestine (Ito et al., 2000). The absorbed MCs were taken up into the hepatocyte and to a lesser extent to other cells by proteins transporters as the multi-specific bile acid transporter (Bury et al., 1998).

Once in the cell, MCs induce toxicity in apparently independent ways. (1) MCs are able to inhibit protein phosphatases which in turn leads to cytoskeletal alterations in liver and kidney (Solter et al., 1998; Codd, 1996; Milutinovic et al., 2003), depletion of glycogen stores (Guzman and Solter, 2002), inmunosupression (Chen et al., 2004) and modify the activities of mitogen-activated protein kinases (MAPK) that support tumour-promoting activities (Pahan et al., 1998) and (2) MCs trigger the production of reactive oxygen species (ROS), that in turn, increase the formation of lipid peroxides (LPO) (Guzman and Solter, 1999; Moreno et al., 2005; Andrinolo et al., 2008), and induce mitochondrial damage, calpain release and apoptosis (Ding and Ong, 2003).

An important effect on hepatocyte morphology produced by prolonged exposure to MCs was the development of cytoplasmic vacuoles in several hepatocites (Solter et al., 1998; Andrinolo et al., 2008). According to this study, a significant increase in hepatic lipid content and in lipid peroxidation levels in liver and kidney tissue were found in MC-LR treated animals when compared with controls (Andrinolo et al., 2008). We hypothesized that an additional hepatotoxic effect of prolonged exposure to MCs is deranged lipid metabolism or induced fatty change, due to the accumulative oxidative damage on lipidic components of the cells.

Both ROS and LPO are known to be reduced by the activity of antioxidant enzymes, such as GPX, GSH-Rd, CAT and SOD (Packer et al., 1998) but little is known about the specific response of antioxidant enzymes to MCs toxicosis. An acute exposure to MC-LR results in a decrease in the antioxidant enzymes and an increase in lipid peroxidation in rats' liver and kidney (Moreno et al., 2005). Recently, a study showed that the antioxidant enzyme system responds differently in organs such as the brain, kidney and liver after a sub-chronic exposure at a lower dosage (Zhao et al., 2009). For these reasons, we also aimed to investigate the changes induced by MC-LR treatment on the antioxidant system in liver and kidney during a sublethal chronic intoxication and also during the recovering (wash-out) period.

Since the outcome of toxicity is determined by the dynamic interplay between damage and its tissue repairing response, we use an experimental model in which evident damage is caused due to the i.p. administration of MC-LR (stage 1) and then recovery or progression of the damage was followed for two months without administration of MC-LR (stage 2). Results obtained may contribute to the understanding of the mechanisms of MC-LR-induced damage on lipid cell composition, the temporal behaviour of the toxicosis, and also the identification of putative therapeutical targets.

\section{Materials and methods}

\subsection{Chemicals}

Pure oxidized and reduced glutathione, $\alpha$-tocopherol, $\mathrm{NAD}^{+}, \mathrm{NADH}(\mathrm{H}), \mathrm{NADPH}(\mathrm{H})$, thiobarbituric acid, N-ethylmaleimide, organic and inorganic components for buffer preparations, sodium pyrophosphate, Tris (tris-hydroxymethyl-aminomethane), ATP (disodium salt), and pyrogallol were purchased from Sigma Chem. Co. (Buenos Aires, Argentina). All solvents used were HPLC grade and provided by Carlo Erba (Milano, Italy). Neutral and polar lipids, and unlabelled fatty acids used as standards were obtained from Serdary Research Lab. (London, Ontario, Canada) or NuCheck-Prep. (Elysian, MN). Other organic and inorganic drugs used were of analytical grade and obtained from Fluka Chemie AG, GmbH \& Co. (Buenos Aires, Argentina).

\subsection{Microcystin-LR purification}

MC-LR was purified from natural blooms of Microcystis aeruginosa collected at the Rio de la Plata basin by established methods (Barco et al., 2005) with slight modifications. Firstly, cells were broken by 3 frozen-unfrozen cycles, and the extract was cut with chloroform/methanol (50:50; v:v). The aqueous fraction was concentrated in a rotovapor (Decalab, R-23, Buenos Aires, Argentina). Finally, the purification was performed with a semipreparative high performance liquid chromatography. We used a Hewlett-Packard apparatus (HP 1100, USA) with a degassed module and a diode array detector system. The preparative column used was TERMO Hyperprep HS $\mathrm{C}_{18}$ $(250 \times 10 \mathrm{~mm})$, and the mobile phase was phosphate buffer ( $\mathrm{pH}$ 7.0) with 30\% acetonitrile, run in isocratic conditions at $5 \mathrm{ml} / \mathrm{min}$. Detection was performed at $238 \mathrm{~nm}$. The peak corresponding to MC-LR was collected separately, concentrated and desalted with a $\mathrm{C}_{18}$ cartridge, previously activated. Pure MC-LR was eluted with a solution of methanol-water $(90: 10, \mathrm{v}: \mathrm{v})$ and the methanol was evaporated. The toxin was tested by HPLC-MS method (Barco et al., 2005). Final identification and concentration of MC-LR were achieved by comparison with a toxin standard from Sigma Chemical Inc. (St. Louis, MO).

\subsection{Mice and treatment}

Twenty four male mice N:NIH-S (20-22 g) with specificpathogen-free certified status were obtained from the Animal Care Facility Unit of the Veterinary Medicine School of La Plata National University. They were housed in plastic cages (four animals in each) and fed ad libitum with balanced food (Alimentos Ganave, Rosario, Argentina) and water. Animals were maintained on a 12-h-light/darknesscycle and allowed to acclimatize to their surrounding conditions (well-ventilated room maintained at $23 \pm 1{ }^{\circ} \mathrm{C}$ ) for 1 week before experiments started. All animals received human care and studies were conducted in accordance with international protocols for laboratory animal care (National Research Council, 1985). Experimental designs were also approved by the local standard for protecting animal's welfare. 


\subsection{Experimental design}

Mice were divided at random into six groups. Treated animals (three groups) were injected $(300 \mu \mathrm{l})$ i.p. every $48 \mathrm{~h}$ during 1 month at a dose of $25 \mu \mathrm{g}$ of MC-LR/kg body weight. The toxin was freshly prepared by dilution of the stock preparation with saline solution $(0.9 \%$; w/v). Control groups of animals were injected with equivalent volume of saline solution. After treatment (30 days for group 1), and after one month (group 2), and two months (group 3) without exposure to the toxin (wash-out periods), mice from the treated and the corresponding control group were slightly anesthetized with ether and they were euthanatized to obtain livers and kidneys. To minimize diurnal variations, animals were routinely killed between 12:00 and 13:00 h. After washing and weighing, both tissues were placed on ice-cold buffered solution (140 mM sodium phosphate, 0.1 mM EDTA, pH 7.40) (Marra et al., 1986), decapsulated, and homogenized by means of a glass-teflon homogenizer (Kontes Glass Company, Vineland, NJ). Cell debris and nuclei were removed by centrifugation at $10,000 \times \mathrm{g}\left(20 \mathrm{~min}\right.$ at $\left.1-2{ }^{\circ} \mathrm{C}\right)$ in a Sorvall RC5C Dupont centrifuge (Newtown, CT). Supernatants were aliquoted and frozen in hermetic polypropylene vials at $-70{ }^{\circ} \mathrm{C}$ under $\mathrm{N}_{2}$ atmosphere until used for experimental measurements. Appropriate aliquots of samples were treated immediately with $40 \mathrm{mM}$ N-ethylmaleimide and precipitated with TCA for glutathione determinations as described in the analytical section.

\subsection{Analytical determinations}

\subsubsection{Lipid analysis}

Individual lipid subclasses were quantified by a densitometric procedure (Marra and de Alaniz, 1992) using the 1D Image Analysis Software, Windows ver. 2.0.3 from Eastman Kodak Company (Rochester, NY). Cholesterol content was enzymatically measured according to Allain et al. (1974). Triacylgliceride (TG) content was assayed using a kit from Wienner Lab. (Rosario, Argentina). Total fatty acid methyl esters (FAMEs) were prepared with $14 \%$ $(\mathrm{W} / \mathrm{V})$ boron trifluoride-methanol following the method of Morrison and Smith (1964) and analyzed by capillary gasliquid chromatography (c-GLC) in a Hewlett Packard HP 6890 Series GC System Plus (Avondale, PA) equipped with a terminal computer integrator. Authentic standards of FAMEs mixtures were routinely run to check the identity of the fatty acids analyzed by means of relative retention times. In some cases, the identity of the fatty acids was confirmed by means of mass-spectrometry analysis (Marra et al., 2002). A standard procedure for a single-step preparation of dimethyl disulfide adducts of the fatty acids was performed according to Yanamoto et al. (1991). Gas-liquid mass-spectrometry analysis of the fatty acid adducts was carried out on a glass column $(1 \mathrm{~m} \times 3 \mathrm{~mm}$ i.d.) packed with 2\% OV-101 on 100-200 mesh Chromosorb WHP (Supleco, Bellefonte, PA) in a Hitachi 663-30 gas chromatograph linked to a Hitachi M-80A Double-focusing Mass Spectrometer (ionization energies from 20 to $70 \mathrm{eV}$ ) with an M-003 minicomputer on-line system. Scan speed was $5 \mathrm{~s}$ per cycle for 0-450 a.m.u. and the column temperature was programmed at $2{ }^{\circ} \mathrm{C} / \mathrm{min}$ from $230{ }^{\circ} \mathrm{C}$ to $260^{\circ} \mathrm{C}$. Perfluorokerosene was used for calibration standard with a resolution power of approx. 6000 .

\subsubsection{Oxidative stress biomarkers}

Contents of oxidized glutathione (GSSG) were determined following the method of Rahman et al. (2006), whereas reduced glutathione (GSH) was measured following the glutathione-S-transferase assay described by Brigelius et al. (1983). Samples for glutathione analyses were obtained in the presence of $\mathrm{N}$-ethylmaleimide and deproteinized ( $1: 9$, by vol.) using trichloroacetic acid (15\% final concentration) as recommended by Asensi et al. (1994). Superoxide dismutase activities (SOD) were assayed as described by Flohé and Ötting (1984). GPX, GSH$\mathrm{Tr}$, and GSH-Rd were assayed according to Wheeler et al. (2001), Habig et al. (1984), and Carlberg and Mannervick (1985), respectively. Catalase activities were measured according to the UV kinetic method of Aebi (1984). $\alpha$ Tocopherol contents were determined by HPLC following the method of Buttris and Diplock (1984). HPLC analyses were performed in a Merck-Hitachi apparatus (Darmstadt, Germany) equipped with an L-6200 Solvent Delivery System and an L-4200 UV/VIS Detector. We used a A$250 \times 4.6 \mathrm{~mm}$ Econosil C-18 column (Alltech Associates, Deerfield, IL) coupled to a $10 \times 4 \mathrm{~mm}$ guard column (packed similarly) run isocratically at $1.5 \mathrm{~mL} / \mathrm{min}$. Nitrate plus nitrite levels (NOx) were assayed by the enzymatic method of Verdon et al. (1995). Protein content was determined by the micromethod of Bradford (1976) with crystalline BSA as standard.

\subsection{Statistical treatment}

All samples were processed individually. The data represent the mean of 4 individual determinations (assayed in duplicate or triplicate) \pm 1 standard deviation (SD). The statistical significance of data was analyzed by either the Student's $t$-test or by ANOVA (analysis of variance) plus Bonferroni test, with the aid of Systat (version 12.0 for Windows) from SPSS Science (Chicago, IL). Data were plotted and analyzed using Sigma Scientific Graphing Software (version 11.0) from Sigma Chem. Co. (St. Louis, MO) and/or GB-STAT Professional Statistics Program (version 4.0) from Dynamic Microsystems Inc. (Silver Springs, USA).

\section{Results}

\subsection{Redox homeostasis}

We first examined the impact of the sub-chronic exposure to MC-LR on the oxidative stress biomarkers in mouse liver and kidney homogenates. Fig. 1 shows the changes observed in the glutathione reductase (GSH-Rd) activity and superoxide dismutase (SOD, total activity) in both tissues. After a 30-day period of MC-LR administration, we observed a significant reduction in the activities of both enzymes not only in liver but also in kidney homogenates. We found an especially diminished SOD activity in kidney preparations after the toxicosis. However, in all cases enzymes were restored to control values after 1 month of 

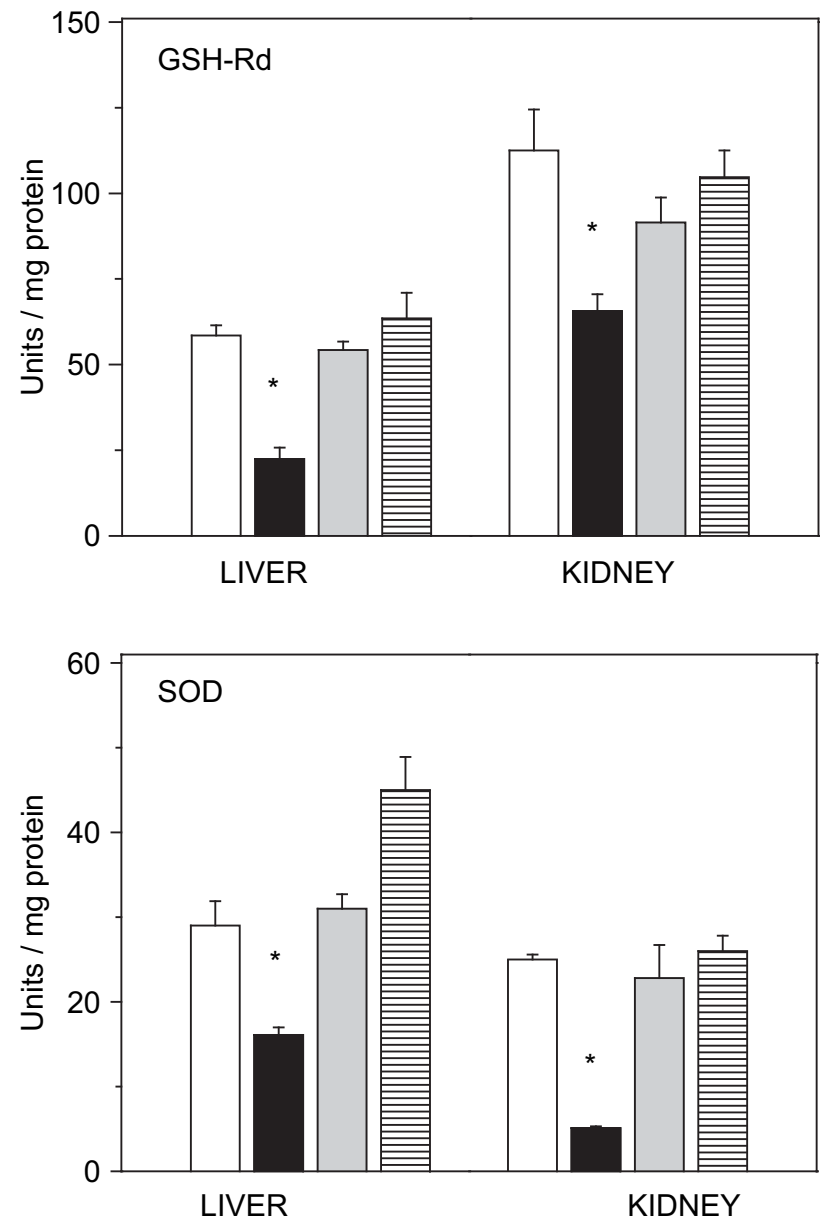

Fig. 1. Glutathione reductase (GSH-Rd) and total superoxide dismutase (SOD) activities in liver and kidney from control (white bars), and exposed animals to MC-LR (25 $\mu \mathrm{g} / \mathrm{kg}$ body weight) during 30 days (black bars), and after 30 (gray bars) or 60 (dashed bars) days toxin suppression. Enzymes were determined as described in Materials and methods section and were expressed (units/mg protein) as the mean \pm SD of four independent determinations assayed in duplicate. *: Significantly different from the corresponding control value $(p<0.01)$

the wash-out period. In the case of the livers, total SOD activity increased significantly compared to control values 2 months after MC-LR deprivation (Fig. 1). Also, GPX and CAT activities were affected by MC-LR administration (Fig. 2). Once again, we found that both enzymes' activity was significantly decreased by MC-LR treatment. After a month without toxin administration the activity of GPX in kidney was restored to control values, while it continued diminishing in liver preparation. CAT activity increased significantly compared to control data after 1 month of the wash-out period in both, liver and kidney homogenates. The activities of both enzymes were normalized after 2 months without MC-LR treatment.

Levels of reduced (GSH) and oxidized (GSSG) glutathione were deeply affected by MC-LR administration in liver and kidney (Fig. 3). We observed that in both tissues GSSG and GSH levels had increased and, as a result, the additional GSH + GSSG also increased compared with control values. The ratio GSH/GSSG was diminished due to the higher increases of GSSG in comparison with those of GSH. After 1 month of the wash-out period, all values
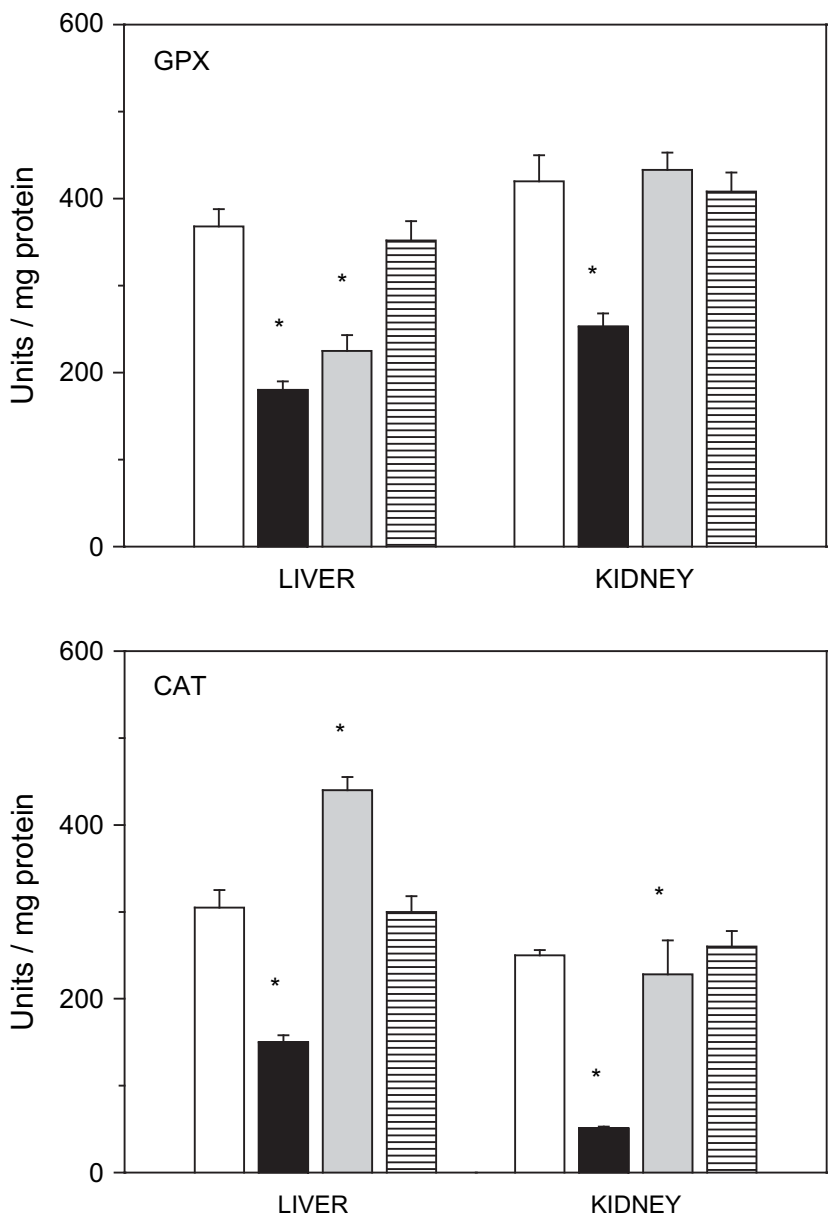

Fig. 2. Glutathione peroxidase (GPX) and catalase (CAT) activities in liver and kidney from control (white bars), and exposed animals to MC-LR (25 $\mu \mathrm{g} /$ $\mathrm{kg}$ body weight) during 30 days (black bars), and after 30 (gray bars) or 60 (dashed bars) days toxin suppression. Enzymes were determined as described in Materials and methods section and were expressed (units/mg protein) as the mean \pm SD of four independent determinations assayed in duplicate. *: Significantly different from the corresponding control value $(p<0.01)$.

returned to control data except the total amount of liver glutathione, which remained significantly elevated (Fig. 3). Interestingly, after 2 months without toxin administration, the only significantly elevated parameter was the ratio GSH/GSSG in kidney. Fig. 4 shows that GSH-Tr activity increased in liver preparations. The increase observed in the kidneys was not statistically significant. After 1 month of toxin suppression the enzyme activity was restored to control values. Estimation of nitric oxide synthetase activity by means of the production of [NOx] (nitrite plus nitrate levels) was increased at the end of the treatment, but it normalized after a 1 month wash-out period. We also observed a significant decrease of $\alpha$-tocopherol in the livers (Fig. 5) that was restored to control values after 1 month of toxin suppression.

\subsection{MC-LR-induced changes on lipid composition}

Fig. 6 shows the changes in the major liver lipid subclasses after injecting MC-LR and during the wash-out period. A significant elevation was observed in some of the 

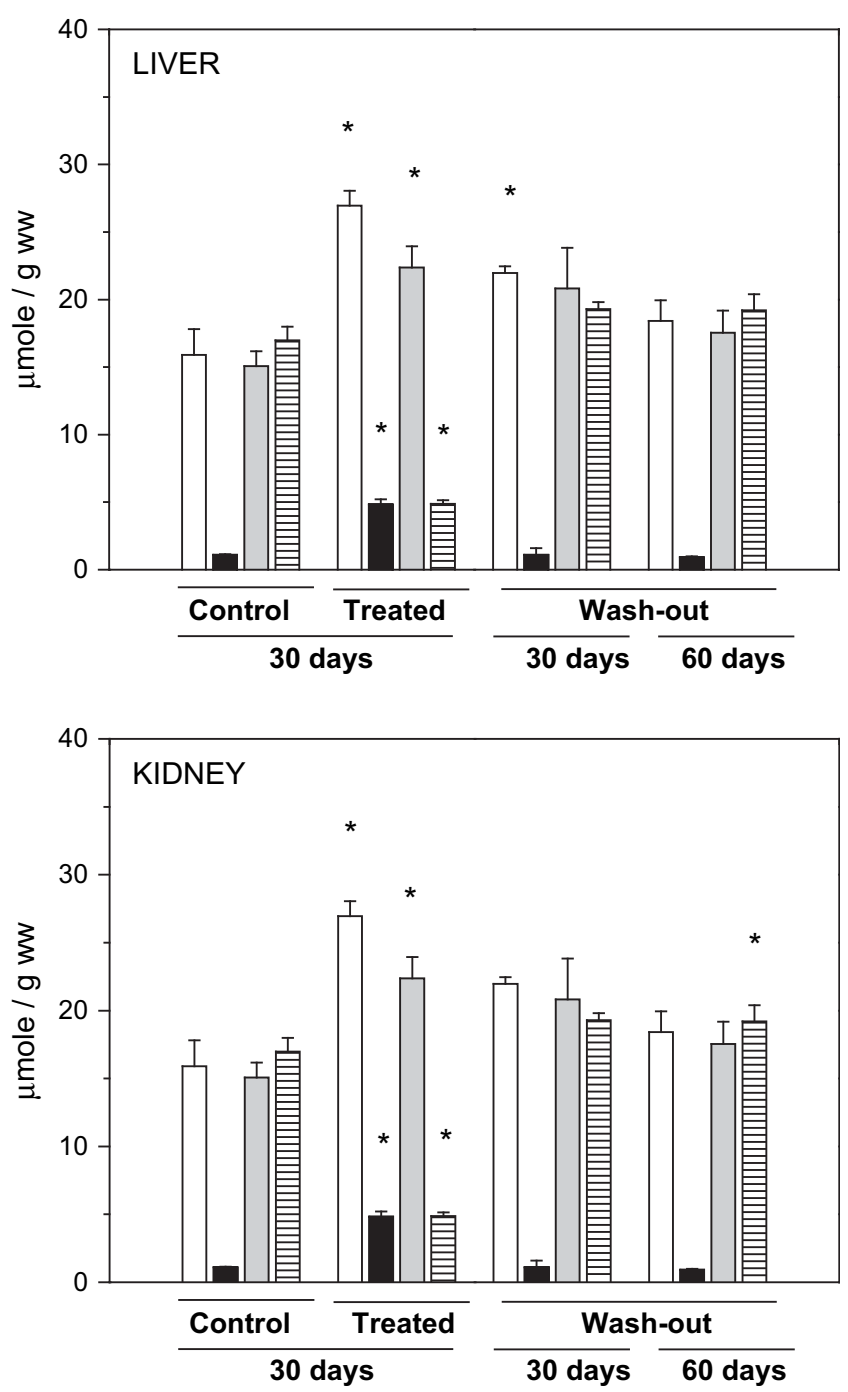

Fig. 3. Total (white bars), reduced (gray bars) and oxidized (black bars) glutathione contents, and GSH/GSSG ratios (dashed bars) in liver and kidney from control, exposed animals to MC-LR ( $25 \mu \mathrm{g} / \mathrm{kg}$ body weight), and toxindeprived mice. GSH and GSSG were determined as described in Materials and methods section and were expressed ( $\mu \mathrm{mol} / \mathrm{g}$ of wet weight) as the mean \pm SD of four independent determinations assayed in triplicate. *: Significantly different from the corresponding control value $(p<0.01)$.

major lipid subclasses studied such as TAG, NEFA, PhtEth and PhtChol. Cholesteryl esthers (ECHO) were significantly reduced.

During the wash-out period, PhtEth level was restored, and a reduction was observed in the elevation for PhtChol. It is interesting to note that the increase induced by MC-LR treatment on TAG levels was maintained, and increased further, after the first month of the wash-out period (Fig. 6). All the parameters normalized after the second month of toxin suppression.

Quantitative main modifications induced by MC-LR treatment on the fatty acyl composition of liver are shown in Table 1. The toxin produced an increment in the absolute quantity ( $\mu \mathrm{mol} / \mathrm{mg}$ protein) of all saturated fatty acids (SFA) such as 18:0, 20:0, 22:0 and 24:0, with a concomitant reduction of monoenoates, especially oleic acid (18:1) and $24: 1$. Within the polyunsaturated fatty acids (PUFA), major changes were observed. Arachidonic acid (20:4 n-6) was significantly reduced together with eicosatrienoate (its

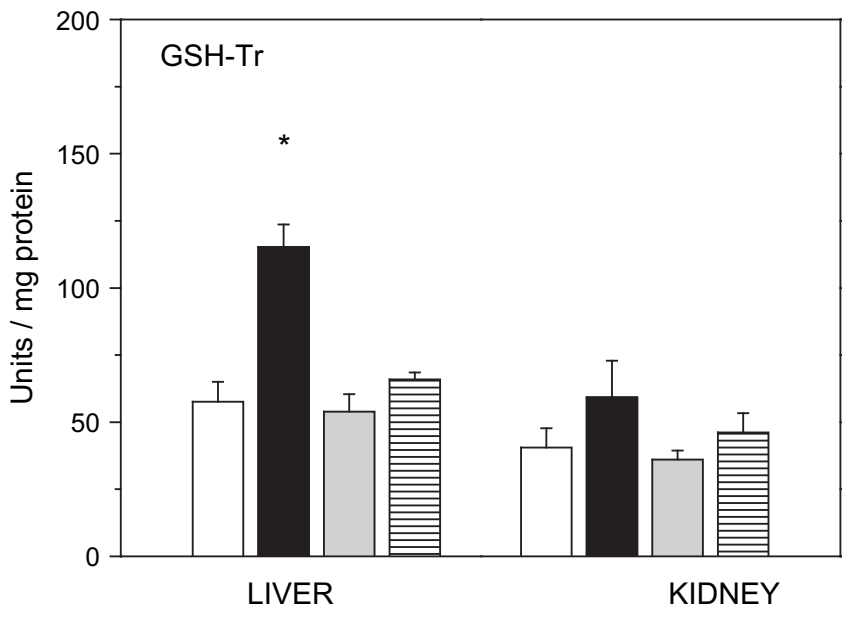

Fig. 4. Glutathione transferase (GSH-Tr) activity in liver and kidney from control (white bars), and exposed animals to MC-LR ( $25 \mu \mathrm{g} / \mathrm{kg}$ body weight) during 30 days (black bars), and after 30 (gray bars) or 60 (dashed bars) days toxin suppression. Enzymes were determined as described in Materials and methods section and were expressed (units/mg protein) as the mean $\pm \mathrm{SD}$ of four independent determinations assayed in duplicate. *: Significantly different from the corresponding control value $(p<0.01)$.

direct metabolic precursor in the n- 6 essential series). At the same time, the 22:3 n-6 level dropped significantly since it derived from the elongation pathway that initiates in 20:3 n-6. Other changes were observed in the quantity of superior homologues of the n-3 series: elevation of 21:5 and reduction in both 22:5 and 22:6. After one month of toxin suppression, all the changes normalized, except those observed for 20:3 and 20:4 of the n- 6 series, $22: 6 n-3$, and 18:0. The elevation in the amount of stearic acid (18:0) was even more evident compared to the values observed after the one-month of toxin treatment (15 and 32\% over control data, respectively). At the end of the second month of the wash-out period, the fatty acyl pattern was completely normalized.

Table 2 shows some analytical parameters calculated from data presented in Table 1. Main changes were observed in the relative proportion of saturated to polyunsaturated fatty acyl chains. The toxin treatment induced a significant reduction of PUFA due to SFA which was directly correlated with a drop in the double bond index (DBI). Even more interesting was the decrease in the ratio n-3/n-6 which was diminished due to a proportionally higher reduction of $n-3$ homologues compared to $n-6$ homologues ( -43 and $-24 \%$ compared to the control data, respectively). Another interesting point was the reduction in the essential to non-essential fatty acid ratio (EFA/nonEFA) since MC-LR administration was able to induce a sort of "EFA deficit" not previously reported for this kind of toxin. Proportions of SFA to PUFA - and consequently DBI remained altered after two months of toxin suppression. However, the relative quantity of $n-3$ to $n-6$ fatty acyl chains had a tendency to normalize after MC-LR suppression.

We also analyzed the analytical parameters involved in the elongation-desaturation routes that start with the conversion of the 18:2 n-6 (linoleic) or 18:3 n-3 (linolenic) essential fatty acids. The metabolic indexes $(\Delta 5+\Delta 6)$ DIA, $\Delta 6$ DIA, and $\Delta 5$ DIA were calculated as described in the 

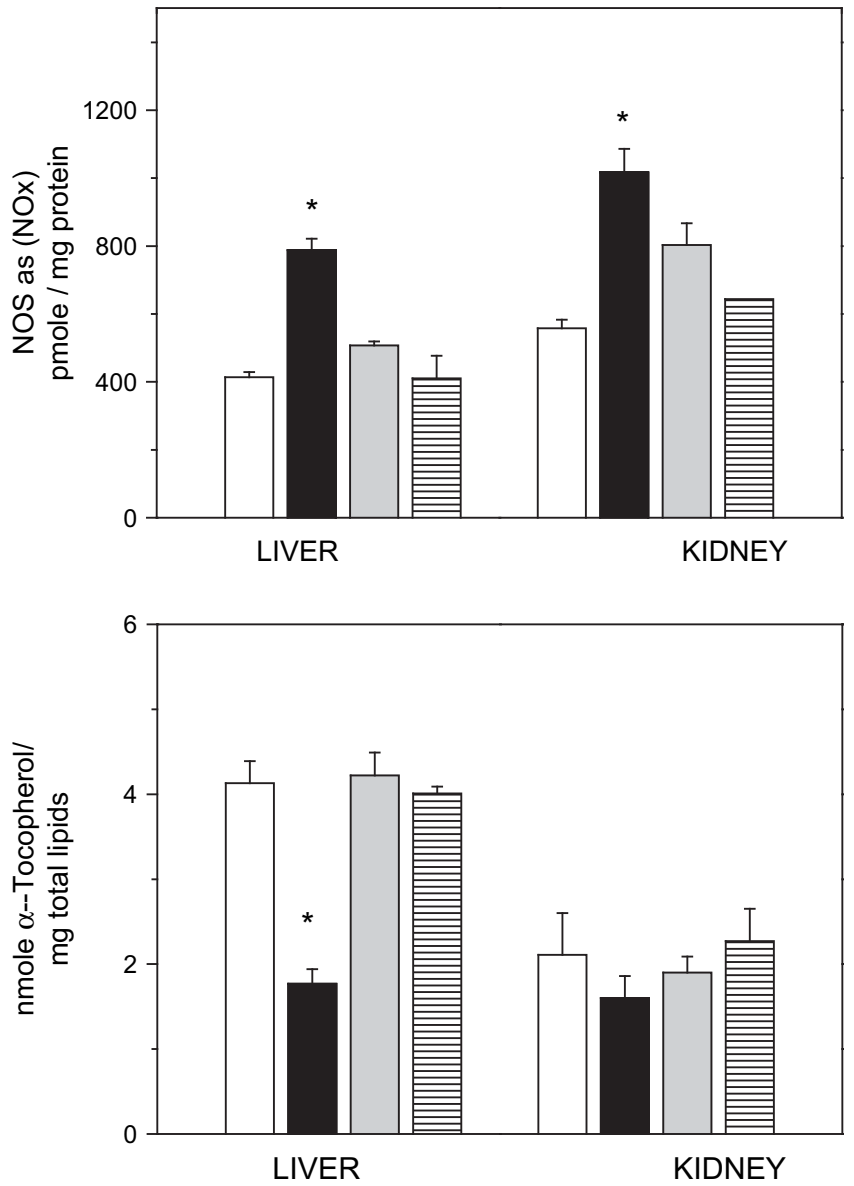

Fig. 5. Nitric oxide synthetase activity (upper panel) estimated as the production of nitrate + nitrite [NOx] levels in liver and kidney from control (white bars), and exposed animals to MC-LR ( $25 \mu \mathrm{g} / \mathrm{kg}$ body weight) during 30 days (black bars), and after 30 (gray bars) or 60 (dashed bars) days toxin suppression. [NOx] was determined as described in the materials and method section and were expressed ( $\mathrm{pmol} / \mathrm{mg}$ protein) as the mean $\pm \mathrm{SD}$ of four independent determinations assayed in triplicate. $\alpha$-Tocopherol content (lower panel) determined in the same preparations detailed before. Results were expressed as nmole of tocopherol per mg total lipids and they were the mean \pm SD of four independent determinations assayed in triplicate. *: Significantly different from the corresponding control value $(p<0.01)$.

legend of Table 2. A significant decrease was observed in $(\Delta 5+\Delta 6)$ DIA at the end of the inflictive stage and at the first month of wash out. $\Delta 6$ DIA showed a significant decrease during the exposure period and a return to control values after the first month of wash out. We observed a significant decrease in $\Delta 5$ DIA index between treated animals and control animals only in the first month of wash out.

Nevertheless, no changes were found in $(\Delta 5+\Delta 6)$ DIA, $\Delta 6$ DIA, and $\Delta 5$ DIA after two month of wash out.

\section{Discussion}

In this study, our aim was to explore the causes of liver and kidney damage induced by chronic treatment with low doses of MC-LR. Our hypothesis is that an additional hepatotoxic effect of MCs is to alter the lipid metabolism or produce fatty change due to the accumulative effect of oxidative damage when the exposure is prolonged.

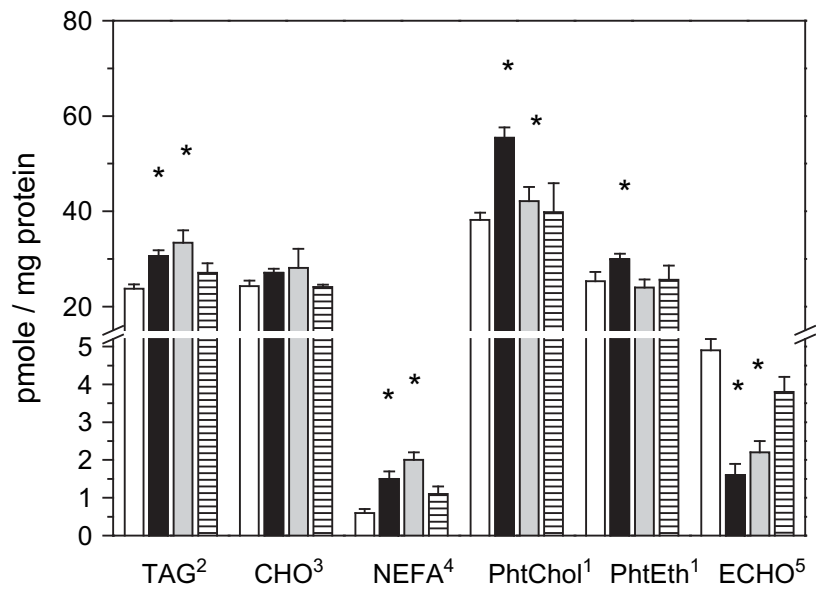

Fig. 6. Major lipid classes in total homogenates from control (white bars), and exposed animals to MC-LR ( $25 \mu \mathrm{g} / \mathrm{kg}$ body weight) during 30 days (black bars), and after 30 (gray bars) or 60 (dashed bars) days toxin suppression. Each sample was collected and separately analyzed under the conditions described in detail in Materials and methods section. Results are the mean of 4 independent determinations assayed in duplicate \pm SD. Results are expressed as: ${ }^{1}$ pmole inorganic phosphate/mg protein, ${ }^{2}$ pmole tripalmitine/mg protein; ${ }^{3}$ pmole/mg protein; ${ }^{4}$ pmole of palmitate/mg protein; ${ }^{5}$ pmole of lynoleyl-cholesterol/mg protein. Significant differences between exposed animals and the corresponding control group were indicated with an asterisk $(p<0.01$ or less).

\subsection{Redox homeostasis}

In agreement with a previous report on acute intoxication in rats (Moreno et al., 2005), and with a prolonged exposure to MCs in rabbits (Zhao et al., 2009) we found decreased levels of CAT, SOD, GPX and GSH-Rd in both liver and kidney preparations from mice treated sub-chronically with MC-LR. Reductions observed for the enzymes were of similar magnitude in both tissues. However, the normalization of the activities revealed a complex interaction between these members of the antioxidant defense system and the non-enzymatic scavengers.

The results of this work supported the idea that a natural consequence of the SOD inhibition is a reduction in the superoxide scavenging. In this way, excess of superoxide may be the responsible for the inhibition of CAT and GPX activity, which in turn, leads to a progressive accumulation of peroxide (Moreno et al., 2005; Andrinolo et al., 2008) and formation of $\mathrm{OH}$. This oxygenated radical is highly reactive against lipids, proteins, and DNA; and there are no scavenging mechanisms to neutralize it (Vranova et al., 2002).

Our results showed that sub-chronic exposure to MC-LR results in an increase in NOx production in kidneys and livers of mice and consequently excessive peroxynitrite formation that may lead to both serious damage in the redox homeostasis and disruption of sensitive biomolecules (especially lipids and nucleic acids) (Packer et al., 1998). Consumption of $\alpha$-tocopherol in liver could be the consequence of this pro-oxidative situation that requires an extra-amount of scavengers in the hydrophobic compartment of the cell.

GPX remains decreased after the first month of the washout period. SOD activity also increased in livers after the second month of wash-out, and CAT was found to have increased in livers and kidneys during the first month of the 
Table 1

Quantitative fatty acyl composition from control or exposed liver homogenates.

\begin{tabular}{|c|c|c|c|c|c|c|}
\hline \multirow[t]{3}{*}{ Fatty acids } & \multirow{2}{*}{\multicolumn{2}{|c|}{$\frac{\text { Treatment }}{30 \text { days }}$}} & \multicolumn{4}{|c|}{ Wash-out periods } \\
\hline & & & \multicolumn{2}{|l|}{30 days } & \multicolumn{2}{|l|}{60 days } \\
\hline & Control & Exposed & Control & Exposed & Control & Exposed \\
\hline $14: 0$ & $0.1 \pm 0.01$ & $0.2 \pm 0.03$ & $0.2 \pm 0.01$ & $0.3 \pm 0.05$ & $0.1 \pm 0.0$ & Traces \\
\hline $15: 0$ & $0.2 \pm 0.02$ & $0.1 \pm 0.0$ & $0.1 \pm 0.0$ & $0.2 \pm 0.03$ & $0.2 \pm 0.03$ & $0.1 \pm 0.01$ \\
\hline $16: 0$ & $21.1 \pm 0.5$ & $23.5 \pm 0.6$ & $19.1 \pm 0.3$ & $22.5 \pm 0.5$ & $20.1 \pm 0.7$ & $19.9 \pm 0.5$ \\
\hline $16: 1(n-9)$ & $2.2 \pm 0.1$ & $2.0 \pm 0.1$ & $1.8 \pm 0.2$ & $2.3 \pm 0.2$ & $1.9 \pm 0.1$ & $2.2 \pm 0.3$ \\
\hline $16: 1(n-7)$ & $0.2 \pm 0.01$ & $0.1 \pm 0.02$ & $0.1 \pm 0.01$ & Traces & $0.2 \pm 0.03$ & $0.3 \pm 0.1$ \\
\hline $17: 0$ & $0.6 \pm 0.03$ & $0.4 \pm 0.04$ & $0.7 \pm 0.1$ & $0.5 \pm 0.1$ & $0.8 \pm 0.1$ & $0.7 \pm 0.2$ \\
\hline $16: 3(n-3)$ & $0.2 \pm 0.01$ & $0.3 \pm 0.0$ & $0.3 \pm 0.05$ & $0.2 \pm 0.03$ & $0.2 \pm 0.0$ & $0.2 \pm 0.05$ \\
\hline $16: 4(n-3)$ & $0.1 \pm 0.0$ & $0.2+0.03$ & $0.3 \pm 0.03$ & $0.2 \pm 0.0$ & $0.4 \pm 0.1$ & $0.3 \pm 0.01$ \\
\hline $18: 0$ & $18.8 \pm 0.7$ & $21.4 \pm 0.6^{*}$ & $17.7 \pm 0.9$ & $23.3 \pm 0.8^{*}$ & $18.5 \pm 0.6$ & $22.2 \pm 0.3$ \\
\hline $18: 1(n-9)$ & $15.1 \pm 0.3$ & $11.5 \pm 0.3^{*}$ & $13.7 \pm 0.1$ & $10.6 \pm 0.4$ & $14.3 \pm 0.3$ & $12.2 \pm 0.3$ \\
\hline $18: 1(n-7)$ & $0.7 \pm 0.1$ & $0.5 \pm 0.1$ & $0.8 \pm 0.1$ & $0.6 \pm 0.1$ & $0.5 \pm 0.1$ & $0.5 \pm 0.05$ \\
\hline $18: 2(n-7)$ & $0.2 \pm 0.0$ & $0.2 \pm 0.04$ & $0.1 \pm 0.03$ & $0.2 \pm 0.03$ & $0.1 \pm 0.0$ & $0.1 \pm 0.03$ \\
\hline $18: 2(n-6)$ & $14.2 \pm 0.3$ & $13.0 \pm 0.4$ & $15.5 \pm 0.5$ & $14.4 \pm 0.6$ & $16.2 \pm 0.3$ & $15.4 \pm 0.5$ \\
\hline $18: 3(n-6)$ & $0.1 \pm 0.03$ & Traces & $0.1 \pm 0.0$ & $0.2 \pm 0.04$ & $0.2 \pm 0.0$ & $0.1 \pm 0.0$ \\
\hline $18: 3(n-3)$ & $0.2 \pm 0.02$ & $0.1 \pm 0.01$ & $0.1 \pm 0.0$ & Traces & $0.1 \pm 0.01$ & $0.2 \pm 0.03$ \\
\hline $18: 4(n-3)$ & $0.1 \pm 0.04$ & Traces & $0.1 \pm 0.02$ & $0.2 \pm 0.03$ & $0.2 \pm 0.03$ & $0.1 \pm 0.01$ \\
\hline $20: 0$ & $0.1 \pm 0.0$ & $0.3 \pm 0.05^{*}$ & Traces & $0.1 \pm 0.01$ & $0.1 \pm 0.02$ & Traces \\
\hline $20: 1\left(\sum n-7+n-9\right)$ & $0.2 \pm 0.01$ & $0.2 \pm 0.02$ & $0.1 \pm 0.03$ & $0.2 \pm 0.0$ & $0.1 \pm 0.0$ & Traces \\
\hline $20: 2(n-6)$ & $0.1 \pm 0.01$ & $0.3 \pm 0.0^{*}$ & $0.1 \pm 0.0$ & $0.1 \pm 0.02$ & Traces & $0.1 \pm 0.03$ \\
\hline $20: 3(n-9)$ & Traces & $0.1 \pm 0.01$ & $0.1 \pm 0.01$ & Traces & $0.1 \pm 0.01$ & Traces \\
\hline $20: 3(n-6)$ & $3.0 \pm 0.1$ & $2.0 \pm 0.2^{*}$ & $2.7 \pm 0.1$ & $2.0 \pm 0.1^{*}$ & $3.1 \pm 0.2$ & $2.8 \pm 0.2$ \\
\hline $20: 4(n-6)$ & $14.1 \pm 0.2$ & $9.0 \pm 0.3^{*}$ & $15.4 \pm 0.6$ & $10.3 \pm 0.4^{*}$ & $14.8 \pm 0.6$ & $13.0 \pm 0.4$ \\
\hline $20: 3(n-3)$ & $0.1 \pm 0.0$ & Traces & Traces & $0.2 \pm 0.05$ & $0.1 \pm 0.0$ & $0.2 \pm 0.02$ \\
\hline $20: 4(n-3)$ & $0.2 \pm 0.03$ & $0.1 \pm 0.01$ & $0.1 \pm 0.02$ & Traces & $0.2 \pm 0.01$ & $0.1 \pm 0.0$ \\
\hline $20: 5(n-3)$ & $0.1 \pm 0.0$ & Traces & $0.2 \pm 0.03$ & $0.1 \pm 0.01$ & $0.1 \pm 0.02$ & Traces \\
\hline $22: 0$ & $0.2 \pm 0.03$ & $0.4 \pm 0.01^{*}$ & $0.1 \pm 0.05$ & Traces & $0.2 \pm 0.05$ & $0.2 \pm 0.02$ \\
\hline $22: 1(n-9)$ & $0.1 \pm 0.0$ & $0.1 \pm 0.0$ & Traces & $0.2 \pm 0.03$ & $0.1 \pm 0.01$ & Traces \\
\hline $21: 5(n-3)$ & Traces & $0.1 \pm 0.03$ & Traces & $0.1 \pm 0.0$ & $0.1 \pm 0.03$ & $0.2 \pm 0.05$ \\
\hline $22: 2(n-6)$ & $0.1 \pm 0.02$ & Traces & $0.1 \pm 0.01$ & Traces & $0.2 \pm 0.03$ & $0.1 \pm 0.0$ \\
\hline $22: 3(n-6)$ & $0.3 \pm 0.02$ & $0.1 \pm 0.02^{*}$ & $0.4 \pm 0.05$ & $0.3 \pm 0.1$ & $0.3 \pm 0.05$ & $0.2 \pm 0.02$ \\
\hline $22: 4(n-6)$ & Traces & Traces & $0.2 \pm 0.01$ & $0.2 \pm 0.05$ & $0.1 \pm 0.0$ & Traces \\
\hline $22: 5(n-6)$ & $0.2 \pm 0.0$ & Traces & $0.1 \pm 0.02$ & Traces & $0.1 \pm 0.02$ & Traces \\
\hline $22: 4(n-3)$ & $0.3 \pm 0.01$ & Traces & $0.4 \pm 0.1$ & $0.2 \pm 0.05$ & $0.2 \pm 0.03$ & $0.2 \pm 0.04$ \\
\hline $22: 5(n-3)$ & $0.5 \pm 0.01$ & $0.1 \pm 0.01^{*}$ & $0.3 \pm 0.02$ & $0.1 \pm 0.03$ & $0.4 \pm 0.1$ & $0.3 \pm 0.05$ \\
\hline $24: 0$ & $0.1 \pm 0.0$ & $0.3 \pm 0.02 *$ & $0.1 \pm 0.0$ & $0.2 \pm 0.04$ & $0.1 \pm 0.0$ & $0.2 \pm 0.04$ \\
\hline $22: 6(n-3)$ & $8.8 \pm 0.3$ & $5.1 \pm 0.2^{*}$ & $9.2 \pm 0.4$ & $6.1 \pm 0.2^{*}$ & $8.9 \pm 0.4$ & $7.0 \pm 0.3$ \\
\hline $24: 1$ & $0.2 \pm 0.0$ & $0.1 \pm 0.03^{*}$ & $0.1 \pm 0.0$ & Traces & $0.2 \pm 0.04$ & $0.2 \pm 0.0$ \\
\hline
\end{tabular}

c-GLC of the fatty acid methyl esters were performed as indicated in Materials and methods section. Samples from control or exposed liver homogenates treated with saline, or toxin solution ( $25 \mu \mathrm{g} / \mathrm{kg}$ body weight), were separately analyzed ( 4 animals each group). Each value is expressed as $\mu$ mol/mg total protein (mean $\pm 1 \mathrm{SD}$ ). Results for the same fatty acid with an asterisk are significantly different $(p<0.01)$ compared to the corresponding control value. Means below $0.1 \%$ are indicated as "traces". Other fatty acids not consigned in the table were present in negligible amounts. SD below 0.01 are indicated as "0.0".

recovery period. These findings may reflect extensive damage in the antioxidant enzymatic system which were unable to neutralize the ROS accumulated (especially superoxide and peroxide) during the exposure period. As a consequence, both tissues required extra activity of the enzymes (SOD and CAT) in order to restore redox homeostasis. It is also conceivable that there could be a sort of inertial deregulation of the enzymes at some point in their genetic expression (Gehringer et al., 2004) that may justify these over-stimulations once the treatment was interrupted.

\subsection{Glutathione pathway}

Toxin-induced oxidative damage had a strong influence on the glutathione metabolism. Glutathione is considered a key non-enzymatic antioxidant within the polar compartment of the cell and constitutes the first line of protection against excessive ROS generation. Some authors have suggested that a loss of reduced glutathione involved in phase II reactions could represent the main route leading the cells to oxidative stress (Moreno et al., 2005). There are two main enzymes involved in the metabolic pathway of glutathione transformation: GPX and GSH-Rd. A previous paper reported that during acute intoxication with MC-LR, GPX and GSH-Tr activities were significantly increased together with an elevation in the total glutathione concentration (Gehringer et al., 2004). In agreement with these results, we also found an increase in the GSH-Tr activity and in the total amount of glutathione. However, the activity of GPX and GSH-Rd were reduced, under subchronic exposure to the toxin. Liver tissue is considered a main tissue involved in GSH biosynthesis and regulation of redox homeostasis and as is well known, the liver is also the target organ for MC-LR intoxication (Falconer, 1994).

It is known that GPX catalyzes the reduction of $\mathrm{H}_{2} \mathrm{O}_{2}$ using GSH. Thus, less availability of reduced glutathione may be the natural consequence for a diminished GPX activity in both tissues (liver and kidney). This result agreed 
Table 2

Analytical parameters from fatty acyl composition of total lipids of control and exposed animals.

\begin{tabular}{|c|c|c|c|c|c|c|}
\hline \multirow[t]{3}{*}{ Parameters } & \multirow{2}{*}{\multicolumn{2}{|c|}{$\frac{\text { Treatment }}{30 \text { days }}$}} & \multicolumn{4}{|l|}{ Wash-out } \\
\hline & & & \multicolumn{2}{|l|}{30 days } & \multicolumn{2}{|l|}{60 days } \\
\hline & Control & Exposed & Control & Exposed & Control & Exposed \\
\hline SFA & $41.2 \pm 0.6$ & $46.6 \pm 0.6^{*}$ & $38.0 \pm 0.9$ & $47.1 \pm 0.8$ & $40.1 \pm 0.7$ & $45.5 \pm 0.5$ \\
\hline PUFA & $42.9 \pm 0.3$ & $30.7 \pm 0.6^{*}$ & $45.8 \pm 0.6$ & $35.1 \pm 0.6$ & $46.1 \pm 0.6$ & $40.6 \pm 0.5$ \\
\hline $\mathrm{DBI}^{\mathrm{a}}$ & $1.04 \pm 0.01$ & $0.66 \pm 0.02$ & $1.20 \pm 0$ & $0.74 \pm 0.02$ & $1.15 \pm 0.1$ & $0.89 \pm 0.2$ \\
\hline Total (n-3) & $10.6 \pm 0.3$ & $6.0 \pm 0.2$ & $11.0 \pm 0.4$ & $7.4 \pm 0.2$ & $10.9 \pm 0.4$ & $8.8 \pm 0.3$ \\
\hline$(n-3) /(n-6)$ & $0.33 \pm 0$ & $0.24 \pm 0$ & $0.32 \pm 0.01$ & $0.57 \pm 0.01$ & $0.31 \pm 0.03$ & $0.27 \pm 0.04$ \\
\hline EFA/non-EFA ${ }^{\mathrm{b}}$ & $0.72 \pm 0.01$ & $0.50 \pm 0.01$ & $0.84 \pm 0.03$ & $0.57 \pm 0.03$ & $0.8 \pm 0.01$ & $0.67 \pm 0.02$ \\
\hline ED $18: 2(n-6)^{c}$ & $1.22 \pm 0.01$ & $0.85 \pm 0.02$ & $1.17 \pm 0.05$ & $0.85 \pm 0.02$ & $1.11 \pm 0.10$ & $1.03 \pm 0.03$ \\
\hline ED $20: 4(n-6)^{d}$ & $0.01 \pm 0$ & - & $0.02 \pm 0$ & $0.02 \pm 0$ & $0.01 \pm 0$ & - \\
\hline$(\Delta 6+\Delta 5) \mathrm{DIA}^{\mathrm{e}}$ & $0.99 \pm 0$ & $0.69 \pm 0.01$ & $0.99 \pm 0.02$ & $0.72 \pm 0.01$ & $0.91 \pm 0.02$ & $0.84 \pm 0.02$ \\
\hline$(\Delta 6) \operatorname{DIA}^{f}$ & $0.21 \pm 0.01$ & $0.15 \pm 0$ & $0.17 \pm 0$ & $0.14 \pm 0.02$ & $0.19 \pm 0.01$ & $0.18 \pm 0.01$ \\
\hline$(\Delta 5) \mathrm{DIA}^{\mathrm{g}}$ & $4.7 \pm 0.11$ & $4.5 \pm 0.05$ & $5.7 \pm 0.14$ & $5.15 \pm 0.21$ & $4.77 \pm 0.15$ & $4.64 \pm 0.10$ \\
\hline PDAI $^{\mathrm{h}}$ & $9.45 \pm 0.32$ & $-^{*}$ & $0.01 \pm 0$ & - & $0.01 \pm 0$ & $0.02 \pm 0$ \\
\hline
\end{tabular}

Results were expressed as the mean \pm 1 SD of 4 independent determinations obtained from data of Table 1 . Calculations for parameters a, $b$, f, h, and i were done according to Lepage et al. (1989); c, and d, were calculated according to Martínez and Ballabriga (1987). * Significantly different respect to the corresponding control values. Amounts below 0.01 are indicated as “-”. SD $<0.01$ is indicated as " 0 ".

a PUFA/saturated.

b $(n-6)+(n-3) /(n-7)+(n-9)+$ saturated.

c $20: 3(n-6)+20: 4(n-6)+22: 5(n-6) / 18: 2(n-6)$.

d $22: 4(n-6)+22: 5(n-6) / 20: 4(n-6)$.

e $20: 4(n-6) / 18: 2(n-6)$.

f $20: 3(n-6) / 18: 2(n-6)$.

g $20: 4(n-6) / 20: 3(n-6)$.

h $16: 1(\mathrm{n}-7) / 16: 0$

i $18: 1(n-9) / 18: 0$.

with previous reports suggesting the close dependence between GPX activity and GSH levels (Gehringer et al., 2004) but was contrary to that expected by Gehringer et al. (2004) which claims that there is evidence that MC-LR to causes an increase in the transcription of GPX. In our findings the GPX remains inhibited during the whole exposure time to MC-LR in kidney and liver. It remains inhibited after a month of wash out in livers (Fig. 3). It was also found that decreased GSH/GSSG levels are directly correlated to diminished enzyme activity.

Another question to be considered from our results is the fact that MC-LR can form a conjugated microcystin-GSH as a sort of a detoxification process catalyzed by GSH-Tr (Pflugmacher et al., 1998). In our experimental system this fact was supported by the elevated levels of GSH-Tr detected after the intoxication period. The conjugation of GSH with MC-LR in an attempt to neutralize its toxicity, also means that there is an important leak of this powerful antioxidant from the available pool, leaving the tissues even more susceptible to oxidative damage (Ding and Ong, 2003; Gehringer et al., 2004). In addition, a higher activity of GSH-Tr in livers compared to kidneys may reflect the main role that liver plays in detoxifying xenobiotics (like MC-LR) through conjugation (Pflugmacher et al., 1998).

A similar pattern was observed for kidneys with the exception of a persistent elevated ratio GSH/GSSG after the second month of toxin suppression. This result may be interpreted as a reestablishment of the reduced capacity of the organ (without new biosynthesis of GSH) once ROS accumulation was compensated. In livers, the equilibrium between GSH and GSSG could be more rapid than in kidneys due to the active metabolism of this antioxidant.

\subsection{MC-LR-induced changes on lipid composition}

We found that the redox dysfunction provoked by MCLR produced extensive damages in the fatty acyl pattern and lipid composition of livers from intoxicated mice. To our knowledge, this is the first time that such changes in lipid composition due to MC-LR sub-chronic intoxication have been reported. A loss of PUFA due to saturated fatty acids should not be surprising due to the decreased sensitivity that SFA showed for ROS-induced peroxidation (Yuan et al., 1999). However, the fact that MC-LR can cause a fatty acid like-deficiency (evidenced by a decreased EFA/nonEFA ratio) was completely unexpected. EFA are important precursors for the biosynthesis of prostaglandins, leukotrienes, tromboxanes, and other autacoids (de Alaniz and Marra, 2003). Thus, MC-LR intoxication could be responsible for alterations in the critical equilibrium among these PUFA metabolites with relevant physiological properties. More surprising was the finding that the proportions between $n-3$ and n-6 fatty acyl chains were differentially affected by the toxin. The decreased level of $n-3$ PUFAs remained altered 30 days after suppression of the toxin. This may be due to the fact that $n-3$ and n-6 PUFAs have a protective (anti-oxidant) effect on oxidative-induced damage (Eder et al., 2006).

We examined the analytical parameters involved in the elongation-desaturation routes starting with the conversion of the 18:2 n-6 (linoleic) or 18:3 n-3 (linolenic) essential fatty acids. The metabolic indexes $(\Delta 5+\Delta 6)$ DIA, $\Delta 6$ DIA, and $\Delta 5$ DIA showed statistic differences between treated and control animals indicating that the changes in the fatty acyl pattern could be a consequence of (1) 
a differential destruction by peroxidation rather than alterations in the biosynthetic pathways, (2) a modification in the fatty acyl elongases and/or desaturases enzymes activities due to a direct or indirect action of the toxin, or (3) a combination of both effects. However, to our knowledge, there are no previous experimental data concerning MC-LR-induced changes on enzymes of the fatty acid metabolism. This possible effect remains to be investigated. Thus, it is likely that the modifications observed on the analytical indexes may result from the destruction of PUFAS by lipid peroxidation under the redox imbalance evoked by the toxin. In addition, analyzing the results of Tables 1 and 2 , a very interesting question is evident. The analytical data that correspond to the wash-out period were completely normalized after two months of toxin suppression while the metabolic indexes remain altered. Thus, these calculations may be relevant in demonstrating that relative rather than absolute alterations between the members of both fatty acid series could be relevant to interpret the damage induced by the MC-LR on cellular lipids.

Our findings also clarify the question why MC-LR toxicosis decreases the fluidity of biological membranes (Hermansky and Stohs, 1991) since this property is directly associated to PUFA contents and DBI values (Valentine and Valentine, 2004).

In conclusion, our results show that chronic exposure to MC-LR generates a deep pro-oxidative situation, with involvement of NO, and an over saturation in the endogenous antioxidant defense system that results in hepatic lipid composition changes. These results invite further research which could find possible therapeutical targets for the treatment of human populations involuntarily expose to the toxin.

\section{Acknowledgements}

The authors are grateful to Norma Cristalli for her excellent technical assistance. The proof reading was performed by Florencia Cobeaga and Colette O'Haire: This study was partially supported by grants from CIC, SECYT PICT 21227-53 and CONICET PIP 6099, Argentina.

\section{Conflicts of interest}

The authors declare no conflict of interest.

\section{References}

Aebi, H., 1984. Catalase in vitro. Methods Enzymol. 105, 121-126.

de Alaniz, M.J.T., Marra, C.A., 2003. Steroid hormones and fatty acid desaturases. Prostaglandins Leukot. Essent. Fatty acids 68, 163-170.

Allain, C.C., Poon, L.S., Chan, C.S., Richmond, W., Fu, P.C., 1974. Enzymatic determination of total serum cholesterol. Clin. Chem. 20, 470-475.

Andrinolo, D., Sedan, D., Telese, L., Aura, C., Masera, S., Giannuzzi, L., Marra, C., Alaniz, M.T., 2008. Recovery after damage produced by subchronic intoxication with the cyanotoxin microcystin LR. Toxicon 51, 457-467.

Asensi, M., Sastre, J., Pallardo, F.V., García de la Asunción, J., Estrela, J.M., Viña, J., 1994. A high-performance liquid chromatography method for measurement of oxidized glutathion in biological samples. Anal. Biochem. 217, 323-328.

Barco, M., Lawton, L.A., Rivera, J., Caixach, J., 2005. Optimization of intracellular microcystin extraction for their subsequent analysis by highperformance liquid chromatography. J. Chromatogr. A 1074, 23-30.
Bradford, M.M., 1976. A rapid and sensitive method for the quantitation of microgram quantities of protein utilizing the principle of protein-dye binding. Anal. Biochem. 72, 248-254.

Brigelius, R., Muckel, C., Akerboom, T.P., Sies, H., 1983. Identification and quantitation of glutathione in hepatic protein mixed disulfides and its relationship to glutathione disulfide. Biochem. Pharmacol. 32, 25292534.

Buttris, J.L., Diplock, A.T., 1984. High-performance liquid chromatography methods for vitamin E in tissues. Methods Enzymol. 105, 131-138.

Bury, N.R., Newlands, A.D., Eddy, F.B., Codd, G.A., 1998. In vivo and in vitro intestinal transport of ${ }^{3} \mathrm{H}$-microcystin-LR, a cyanobacterial toxin, in rainbow trout (Oncorhynchus mykiss). Aquat. Toxicol. 42, 139-148.

Carlberg, I., Mannervick, B., 1985. Glutathione reductase. Methods Enzymol. 113, 484-490.

Chen, T., Zhao, X., Liu, Y., Shi, Q., Hua, Z., Shen, P., 2004. Toxicology analysis of immunomodulating nitric oxide, iNOS and cytokines mRNA in mouse macrophages induced by microcystin-LR. Toxicology 197, 67-77.

Codd, G.A., 1996. Mechanism of action and healt effects associated with cyanobacterial toxins. Toxicol. Lett. 88, 21.

Ding, W., Ong, C., 2003. Role of oxidative stress and mitochondrial changes in cyanobacteria-induced apoptosis and hepatotoxicity. FEMS Microbiol. Lett. 220, 1-7.

Eder, E., Wacker, W., Lutz, U., Nair, J., Fang, X., Bartsch, H., Beland, F.A., Schlatter, J., Lutz, W.K., 2006. Oxidative stress related DNA adducts in the liver of female rats fed with sunflower-, rapeseed-, olive- or coconut oil supplemented diets. Chem. Biol. Interact. 159, 81-89.

Falconer, I., 1994. Mechanism of Toxicity of Cyclic Peptide Toxins from Blue-green Algae. In: Falconer, I. (Ed.), Algal Toxins in Seafood and Drinking Water. Academic Press, Cambridge, pp. 177-187.

Falconer, I.R., 1999. An overview of problems caused by toxic blue-green algae (cyanobacterial) in drinking and recreational waters. Environ. Toxicol. 14, 5-12.

Flohé, L., Ötting, F., 1984. Superoxide dismutase assays. Methods Enzymol. 105, 93-104.

Gehringer, M.M., Shepard, E.G., Downing, T.G., Wiegand, C., Neilan, B.A., 2004. An investigation into the detoxification of MC-LR by the glutathione pathway in Balb/c mice. Int. J. Biochem. Cell. Biol. 36, 931-941.

Guzman, R.E., Solter, P.F., 1999. Hepatic oxidative stress following prolonged sublethal microcystin LR exposure. Toxicol. Pathol. 5, 582-588.

Guzman, R.E., Solter, P.F., 2002. Characterization of sublethal microcystinLR exposure in mice. Vet. Pathol. 39, 17-26.

Habig, W.H., Pabst, M.J., Jakoby, W.B., 1984. Glutathione-S-transferases. The first enzymatic step in mercapturic acid formation. J. Biol. Chem. 249, 7130-7139.

Hermansky, S.J., Stohs, S.J., 1991. Effect of MC-LR (MCLR) on hepatic microsomal membrane fluidity. Res. Comm. Chem. Pathol. Pharmacol. $72,213-222$.

Ito, E., Kondo, F., Harada, K., 2000. First report on the distribution of orally administered microcystin-LR in mouse tissue using an immunostaining meted. Toxicon 38, 37-48.

Jochimsen, E.M., Carmichael, W.W., An, J.S., Cardo, D.M., Cookson, S.T., Holmes, C.E.M., Antunes, M.B.D., Melo, D.A., de Lyra, T.M., Barreto, V.S. T., Azevedo, S., Jarvis, W.R., 1998. Liver failure and death after exposure to microcystins at a hemodialysis center in Brazil. New Engl. J. Med. 338, 873-878.

Lepage, G., Levy, E., Ronco, N., Galeano, N., Roy, C., 1989. Direct trasnsesterification of plasma fatty acids for the diagnosis of essential fatty acid deficiency in cystic fibrosis. J. Lipid Res. 30, 1483-1490.

Marra, C.A., de Alaniz, M.J.T., Brenner, R.R., 1986. Modulation of $\Delta 6$ and $\Delta 5$ rat liver microsomal desaturase activities by dexamethasone-induced factor. Biochim. Biophys. Acta 879, 388-393.

Marra, C.A., de Alaniz, M.J.T., 1992. Incorporation and metabolic conversion of saturated and unsaturated fatty acids in SK-Hep1 human hepatoma cells in culture. Mol. Cell. Biochem. 117, 107-118.

Marra, C.A., Zaidenmerg, A., de Alaniz, M.J.T., Buschiazzo, H., 2002. The restoring effect of trifluralin and benznidazole on the abnormal fattyacid pattern induced by Trypanosoma cruzi in the liver microsomes of infected mice. Ann. Trop. Med. Pharmacol. 96, 249-264.

Martínez, M., Ballabriga, A., 1987. Effect of parenteral nutrition with high doses of linoleate on the developing human liver and brain. Lipids 22 , 133-138.

Milutinovic, A., Zivin, M., Zorc-Pleskovic, R., Sedmak, B., Suput, D., 2003. Nephrotoxic effects of chronic administration of microcystins-LR and -YR. Toxicon 42, 281-288.

Moreno, I., Pichardo, S., Jos, A., Gómez-Amores, L., Mate, A., Vazquez, C.M., Cameán, A.M., 2005. Antioxidant enzyme activity and lipid peroxidation in liver and kidney of rats exposed to MC-LR administered intraperitoneally. Toxicon 45, 395-402. 
Morrison, W.R., Smith, L.M., 1964. Preparation of fatty acid methyl esters and dimethylacetals from lipids with boron fluoride-methanol. J. Lipid Res. 5, 600-608.

National Research Council, 1985. Guide for the Care and Use of Laboratory Animals. Publication No. 85-23 (rev). National Institute of Health, Bethesda, MD.

Pahan, K., Sheikh, F., Namboodiri, A., Singh, I., 1998. Inhibitors of protein phosphatase 1 and $2 \mathrm{~A}$ differentially regulate the expression of inducible nitric-oxide synthase in rat astrocytes and macrophages. JBC 273 (15), 12219-12226.

Packer, M.A., Scarlett, J.L., Martin, S.W., 1998. Peroxynitrite: a biologically significant oxidant. Gen. Pharmacol. 31, 179-186.

Pflugmacher, S., Weigand, C., Oberemm, A., Beattie, K.A., Krause, E. Codd, G.A., Steinberg, C.E.W., 1998. Identification of an enzimatically formed glutathione conjugated of the cyanobacterial hepatotoxin MC-LR: the first step of detoxication. Biochim. Biophys. Acta 1425, 527-533.

Rahman, T., Kode, A., Biswas, S.K., 2006. Assay for quantitative determination of glutathione and glutathione disulfide levels using enzymatic recycling method. Nat. Protoc. 1, 3159-3165.

Solter, P., Wollenberg, G., Huang, X., Chu, F., Runnegar, M., 1998. Prolonged Sublethal Exposure to the Protein Phosphatase Inhibitor MC-LR Results in Multiple Dose-Dependent Hepatotoxic Effects. Toxicol. Sci. 44, 87-96.

Ueno, Y., Nagata, S., Tsutsumi, T., Hasegawa, A., Watanabe, M.F., Park, H.D. Chen, G.C., Chen, G., Yu, S.Z., 1996. Detection of microcystins, a bluegreen algal hepatotoxin, in drinking water sampled in Haimen and
Fusui, endemic areas of primary liver cancer in China, by highly sensitive immunoassay. Carcinogenesis 17, 1317-1321.

Valentine, R.C., Valentine, D.L., 2004. Omega-3 fatty acids in cellular membranes: a unified concept. Prog. Lipid Res. 43, 383-402.

Verdon, C.P., Burton, B.A., Prior, R.L., 1995. Sample pretreatment with nitrate reductase and glucose-6-phopshate dehydrogenase quantitatively reduces nitrate while avoiding interference by $\mathrm{NADP}^{+}$when the Griess reaction is used to assay for nitrite. Anal. Biochem. 224, 502-508.

Vranova, E., Inzé, D., Van Breusegem, F., 2002. Signal transduction during oxidative stress. J. Exp. Bot. 53, 1227-1236.

Wheeler, M.D., Nakagami, M., Bradford, B.U., Uesugi, T., Mason, R.P. Connor, H.D., Dikalova, A., Kadiiska, M., Thurman, R.G., 2001. Overexpression of manganese superoxide dismutase prevents alcoholinduced liver injury in the rat. J. Biol. Chem. 276, 36664-36672.

WHO, 2004. Guidelines for Drinking-Water Quality. Recommendations. Chemical Fact Sheets, third ed., vol. 1. World Health Organization, Geneva, Switzerland, pp. 407-408.

Yanamoto, K., Shibahara, A., Nakayama, T., Kajimoto, G., 1991. Determination of double-bond positions in methylene-interrupted dienoic fatty acids by GC-MS as their dimethyl disulfide adducts. Chem. Phys. Lipids 60, 39-50.

Yuan, Y.V., Kitts, D.D., Godin, D.V., 1999. Influence of increased saturated fatty acid intake from beef tallow on antioxidant status and plasma lipids in atherosclerosis-susceptible Japanese quail. Nutr. Res. 19, 461-481.

Zhao, Y., Xie, P., Zhang, X., 2009. Oxidative stress response after prolonged exposure of domestic rabbit to a lower dosage of extracted microcystins. Environ. Toxicol. Pharmacol. 27, 195-199. 